Meta

Journal des traducteurs

Translators' Journal

\title{
La retraduction, retour et détour
}

\section{Yves Gambier}

Volume 39, numéro 3, septembre 1994

URI : https://id.erudit.org/iderudit/002799ar

DOI : https://doi.org/10.7202/002799ar

Aller au sommaire du numéro

Éditeur(s)

Les Presses de l'Université de Montréal

ISSN

0026-0452 (imprimé)

1492-1421 (numérique)

Découvrir la revue

Citer cet article

Gambier, Y. (1994). La retraduction, retour et détour. Meta, 39(3), 413-417.

https://doi.org/10.7202/002799ar d'utilisation que vous pouvez consulter en ligne.

https://apropos.erudit.org/fr/usagers/politique-dutilisation/ 


\title{
LA RETRADUCTION, RETOUR ET DÉTOUR
}

\author{
YVES GAMBIER
}

Université de Turku, Turku, Finlande

En hommage à Antoine Berman

\begin{abstract}
Dostoïevski, Kafka, M. Lowry, Kundera, Freud, Hegel... ont été retraduits (en français) ou sont en train de l'être. Les versions antérieures étaient-elles fautives? Pourquoi a-t-on attendu pour les revoir?

Nos connaissances du russe, de l'allemand... ont-elles été modifiées? Lit-on, interprète-t-on autrement des auteurs pourtant placés parmi les valeurs universelles? La notion de fidélité a-t-elle connu un glissement de sens?

Les questions ne manquent pas devant ces travaux refaits, souvent justifiés parce que les premières traductions sont perçues désormais comme entachées de tics francofrançais, que les originaux ont vraiment été (trop) arrangés, apprivoisés aux normes du bon français... Simple toilettage alors qui expliquerait le peu de cas qu'on fait en général de ce phénomène - $\mathrm{y}$ compris en traductologie?
\end{abstract}

\section{RETRADUIRE OU PAS}

La retraduction serait une nouvelle traduction, dans une même langue, d'un texte déjà traduit, en entier ou en partie. Elle serait liée à la notion de réactualisation des textes, déterminée par l'évolution des récepteurs, de leurs goûts, de leurs besoins, de leurs compétences... À noter cependant que le Grand Robert (édition de 1985) préfère attribuer à ce terme le sens de «traduction d'un texte lui-même traduit d'une autre langue»: la retraduction serait donc l'étape ultime d'un travail réalisé grâce à un intermédiaire, à un texte-pivot. Cette deuxième traduction - ou traduction de traduction - n'est pas rare : elle permet l'accès à des langues-cultures peu répandues - par exemple un ouvrage en arabe égyptien rendu en finnois via une version anglaise, un film indonésien sous-titré via un dialogue déjà adapté en une langue étrangère... Autre cas : l'interprétation simultanée par relais (p. ex.: grec-français-portugais).

Ces deux types de retraduction se distinguent encore de la rétrotraduction, qui consiste à traduire de nouveau une traduction vers sa langue de départ, à replonger un texte à sa source pour vérifier les correspondances, la validité des choix opérés par le traducteur (p. ex. : traduction anglaise d'un texte allemand, retraduit en allemand).

Enfin, en abordant le phénomène de retraduction, on ne peut manquer de s'interroger sur ses rapports à la révision et à l'adaptation, elles-mêmes problématiques quant à leur extension. N'y aurait-il entre les trois qu'une différence de degrés dans les retouches / transformations apportées au texte traduit? On aurait alors comme un continuum du moins vers le plus: de la révision (peu de modifications) vers l'adaptation (tant de modifications que l'original peut être ressenti comme un prétexte à une rédaction autre), en passant par la retraduction (beaucoup de modifications, telles que c'est presque entièrement tout le texte qu'il faut revoir). On notera que si les trois types prétendent viser à une communication plus efficace, seule la retraduction conjugue à cette dimension socio-culturelle la dimension historique : elle apporte des changements parce que les temps ont changé. Il resterait à creuser davantage les rapprochements : s'agit-il d'un effort à l'autre des mêmes modifications, des mêmes niveaux de transformation? Un ajustement syntaxique, stylistique... en révision a-t-il le même poids, la même fréquence qu'en retraduction? 
C'est dire déjà qu'au-delà des apparences, le concept de retraduction reste à approfondir - y compris dans sa dimension commerciale: la nouvelle traduction est-elle un argument de vente alors qu'une traduction révisée est moins chère?

La retraduction appelle nombre de questions - peut-être parce qu' «elle est un genre polymorphe dont la multiplicité potentielle provient du polymorphisme de la fonction, des fonctions, du traducteur» (Rodriguez 1990: 65):

- Pourquoi un même texte suscite-t-il plusieurs traductions? Il s'agit souvent de textes littéraires mais pas exclusivement: les textes religieux (la Bible, par exemple), philosophiques (Platon, Leibniz, Hegel...), en sciences sociales (Durkheim, Marx, Weber...), en histoire, en psychanalyse... connaissent aussi de secondes traductions.

- Pourquoi certaines traductions vieillissent-elles vite tandis que d'autres perdurent? Les premières sont-elles davantage de type cibliste, centrées sur les normes de la langue-culture d'arrivée, tandis que les autres seraient plutôt de type sourcier, littérales ( $c f$. Pasanen 1990, à propos de la King James Version de la Bible, achevée en 1611)?

- La retraduction se pose-t-elle dans les mêmes termes, avec la même acuité selon les genres - roman à la première personne, récit polyphonique jouant de divers registres de langue (Gresset 1990), poésie en prose ou en vers...? Dans le cas des pièces de théâtre (Benhamou 1990), on parle souvent d'adaptation (Gambier 1992): la retraduction s'applique-t-elle alors?

m. Qu'en est-il des autotraductions (Strindberg, Nabokov, J. Green, Beckett...), des textes multilingues (J. Joyce, E. Pound...) : sont-ils retraduisibles et à quelles conditions?

- D'une traduction à l'autre, quel est le rôle majeur, dominant que joue le traducteur (Rodriguez 1990) ? Est-il tantôt le traître à son auteur, le serviteur de ses lecteurs, tantôt le messager de l'autre, de la différence au détriment des conventions de réception? Si finalement la question de la retraduction n'est encore que peu posée, c'est que sans doute celle de la traduction n'a pas fini de se poser, depuis au moins le XVI ${ }^{\mathrm{e}}$ siècle.

\section{RETRADUCTION ET INTRADUISIBILITÉ}

La retraduction travaille sur des textes déjà introduits en langue-culture d'arrivée. La relation que le traducteur établit avec ces premières versions mériterait une double analyse, fondée sur les écrits eux-mêmes et sur les opinions des traducteurs, des éditeurs, des lecteurs - de manière à saisir jusqu'où il y a détachement par rapport à la première traduction et comment les différences d'une traduction à la suivante sont perçues. Faute de cette double analyse, on en est encore réduit à faire des hypothèses, des supputations.

Ainsi, à la suite de Berman (1986 et 1990), on peut prétendre qu'une première traduction a toujours tendance à être plutôt assimilatrice, à réduire l'altérité au nom d'impératifs culturels, éditoriaux : on fait des coupures, on réarrange l'original au nom d'une certaine lisibilité, elle-même critère de vente. La retraduction dans ces conditions consisterait en un retour au texte-source. Il peut y avoir, dans ce processus, illusion en partie: ne supposerait-on pas en effet que le sens est déposé dans le texte de départ considéré comme immuable? Cette vision logocentrique du texte et de l'immanence du sens marque d'une certaine façon, par exemple, le projet de retraduction de Freud - comme si les traducteurs pouvaient se débarrasser des interprétations postérieures à l'œuvre même, comme s'ils pouvaient faire une lecture non idéologique, non culturelle d'un sens prétendument stable. L'aveuglement des premiers traducteurs serait dépassé par les dénégations des seconds, dans leur effort pour se rapprocher de la source. En fait, ce rapprochement lui-même est historiquement marqué : il n'est possible justement que parce qu'il y a déjà eu première traduction. La retraduction est un retour dévoyé, indirect : on ne peut tenter 
une traduction autre qu'après une période d'assimilation qui permet de juger comme inacceptable le premier travail de transfert. Proust, par exemple, a pu être retraduit en anglais parce qu'il y a eu nouvelle édition (complétée) des textes et parce que l'écriture proustienne a pris place dans les formes littéraires admises. La retraduction n'est pas découverte au sens trivial du mot; elle l'est néanmoins dans son effort de rapprochement littéral : elle découvre une écriture recouverte par les normes et conventions de la langue d'arrivée. S'il y a retour, c'est par le détour de la première traduction, elle-même souvent ouvrage de détournement. La retraduction délie les formes asservies, restitue la signifiance, ouvre aux spécificités originelles, tout en faisant travailler la langue traduisante : retraduire Apollinaire en finnois, c'est percevoir enfin que le poète n'est pas Finlandais, n'est pas inscrit dans la tradition locale.

La retraduction apporte des réponses nouvelles aux questions toujours posées consciemment ou pas par le traducteur: qu'est-ce que je comprends? (cf. l'objection préjudicielle de Ladmiral (1979)) qu'est-ce que je peux traduire? (cf. la quodité traductive (Ladmiral 1979)). Ces réponses qui donnent des frontières différentes à l'intraduisibilité sont tributaires de divers facteurs telles une édition renouvelée de 1'original, une évolution dans les moyens d'interprétation - grâce à l'analyse génétique des manuscrits, aux nouveaux outils théoriques de la littérature, aux approches sociologiques de la réception, etc. - une conscience linguistique différente qui accorde un statut autre aux langues en présence, qui ressent différemment les variations et les niveaux de langue, qui définit de nouveau l'acceptabilité de telles formes, de tels mots, de telles tournures...

Dans ces conditions, que retraduit-on?

Il y a les retraductions visibles qui portent sur des parties initialement supprimées, allégées, sur des passages naguère amputés, censurés... Des retraductions peuvent ainsi être partiellement des premières traductions...

Il y a les retraductions qui portent sur des contresens (p. ex. : La plaisanterie de Kundera), sur des allusions mises à jour. Et puis celles qui remédient à la lourdeur du style de la ou des traductions antérieures, qui (re)donnent le ton, le rythme de l'original... Les éléments retraduits - venant d'une édition plus complète, des exigences des récepteurs, de la relecture du traducteur... - permettent-ils de définir une stratégie particulière de la retraduction? On retrouve ici la question du rapport du traducteur aux versions qui le précèdent, faites en des temps - sinon des lieux — différents pour des lecteurs différents : pourtant, s'il vient après la première traduction, il ne travaille pas forcément à partir d'elle. À cela s'ajoute son rapport à l'original : sur quelle édition se penche-t-il? Quel est l'éloignement dans le temps de cet original ? Ce dernier point est à traiter d'autant que la première traduction a pu avoir lieu peu après la sortie du texte de départ, c'est-à-dire dans des conditions et avec des contraintes inconnues de la retraduction.

\section{AVEC LE TEMPS...}

Activité soumise au temps - temps de la réception, durée du processus même, acceptabilité datée du produit du transfert, la traduction est un acte toujours inachevé, à refaire. Mais toutes les traductions ne vieillissent pas à la même allure, au même degré. Cette «temporalité de la caducité et de l'inachèvement» (Berman 1990: 1) n'atteint pas toutes les traductions de la même manière: certaines transcendent leur propre historicité — ce sont les «grandes traductions» (Berman 1990: 3-4), nécessairement retraductions - parmi lesquelles on peut citer la Bible de Luther, le Plutarque d'Amyot, la version des Mille et une nuits de Galland, 1'Homère de Pope, le Shakespeare de Schlegel ou de Tieck, l'Antigone de Hölderlin, le Milton de Chateaubriand, le Poe de Baudelaire...

A la fois appropriation et expropriation langagière, la grande traduction rendrait manifestes les différences entre langue-texte de départ et langue-texte d'arrivée: au lieu 
d'occulter le travail de transfert, elle le donnerait à voir, à lire dans la tension même du contact interlinguistique. Elle se définirait par la mise en texte de l'étrangeté. Marquées par les axiomes de l'équivalence dynamique, de la bonne traduction comme celle qui ne sent pas la traduction..., les théories actuelles de la traduction (p. ex. Nida, Vermeer, Seleskovitch...) n'aident guère à comprendre, à expliquer le phénomène de la retraduction, les réussites des grandes traductions. Elles ignorent encore d'ailleurs les conditions et les contraintes d'une histoire de la traduction.

Même si cette histoire reste à faire, bien des exemples de retraduction (vers le français) viennent à la mémoire qui mériteraient une observation fouillée: Virgile - si traduit au $\mathrm{XVI}^{\mathrm{e}}$ puis au XVIII ${ }^{\mathrm{e}}$ siècle et jusqu'en 1964 -, Dante, Shakespeare, Dickens, Swift...

On pourrait alors répondre aux questions suivantes:

- sur la périodicité et la fréquence des retraductions;

- sur les moments aigus de reprise de textes, d'auteurs - c'est-à-dire de moindre résistance ou de plus grande ouverture de la langue-culture d'accueil ;

- sur les motifs, les décisions de retraduire et les transformations de la langue, de la culture, de la littérature réceptrices (avec les théories du discours induites par ces transformations);

- sur les stratégies, les enjeux et les effets de ces nouvelles traductions;

- sur leurs caractéristiques communes, s'il en est - notamment au regard des conceptions de l'équivalence, de la fidélité, de la liberté en traduction.

Pour en revenir au problème de la temporalité, une question ne peut pas ne pas être posée maintenant: le rapport au temps est-il similaire pour l'original et sa traduction? $\mathrm{La}$ notion de décalage temporel a-t-elle le même sens pour les deux? On a vu qu'une traduction ordinaire, figée dans les normes d'une époque, date vite. Un texte source, au contraire, ne semble pas prendre de l'âge - mais là aussi, il faut distinguer, semble-t-il, entre les textes: entre ceux qui vont dans le sens de la rhétorique établie, qui la reproduisent (parfois best-sellers, vite remisés dans l'oubli) et ceux qui innovent, transgressent les habitudes de lecture, d'écriture. Un même texte peut d'ailleurs porter en lui ces deux aspects, conservateur et novateur ( $c f$. certains récits de Kafka, de Joyce, p. ex.). On ne peut donc opposer de façon tranchée original et traduction, dans leur rapport au temps (cf. Topia 1990: 45-49). Tous les originaux ne sont pas des chefs-d' $d^{\prime} u v r e$, comme toutes les retraductions ne sont pas de grandes traductions. Il y a des originaux — justement sans originalité - comme des traductions qui ne font pas long feu, pris dans leur dépendance aux canons dominants; il y a des chefs-d' œuvre comme de grandes traductions qui font date dans l'histoire des échanges culturels et littéraires. Cette grandeur n'est pas que définition institutionnelle, reflétée dans les Académies, les Panthéons, les anthologies, les auteurs aux programmes scolaires... elle est cet effort pour faire bouger les formes, pour féconder la langue, pour réajuster les réseaux de sens, les jeux de l'intertextualité.

Un travail sur corpus reste à faire pour mieux cerner le concept de retraduction et la place finalement des traductions dans une société donnée: celles-ci ne sont pas simples textes secondaires, en rapport unique avec le seul sens des textes originaux — une telle perception orientée vers la source, sinon bornée à elle, revient souvent au même qu'une approche normative, aveugle aux fonctions (économique, idéologique, littéraire) remplies par la traduction. Toute stratégie traductionnelle implique un projet, un pacte, un contrat, c'est-à-dire une réponse aux questions: qui traduit?, pourquoi et quoi ?, avec quelles intentions, déclarées ou pas? Réalisée dans et par un ensemble de décisions, la traduction doit à la fois servir l'auteur ET être au service de ses lecteurs. C'est cette double allégeance qui fait l'originalité du travail du traducteur, en symbiose avec le texte à traduire et les traditions de sa communauté, possédé par ses langues et apte à les subvertir. 
En réfléchissant sur la retraduction, un dernier exemple vient à la pensée: celui des originaux qui se donnent comme traductions (pseudotraductions, traductions fictives) - procédé assez courant dans l'histoire littéraire... Qu'on se rappelle Cervantès (Don Quichotte), Montesquieu (Les Lettres persanes), Voltaire (Candide), Mérimée, Pierre Loiiys, B. Vian (J'irai cracher sur vos tombes), Tolkien, Borges, L. Sciascia, J. Cela, U. Eco (Le nom de la rose), etc. Comment retraduit-on ces textes, dans le double sens du mot ?

Ces lignes ont été rédigées quasiment au moment de la mort d'A. Berman (22 novembre 1991). Elles lui sont largement redevables. Aussi lui sont-elles dédiées - en hommage à sa largeur d'esprit et à son non-conformisme.

\section{RÉFÉRENCES}

ATLAS (1988): $4^{\mathrm{e}}$ Assises de la traduction littéraire à Arles, 7-9 novembre 1987, pp. 66-88 des Actes, Actes Sud (trois ateliers sur la retraduction, avec l'anglais, l'allemand, le portugais).

ATLAS (1989): $5^{\mathrm{e}}$ Assises, 11-13 novembre 1988, pp. 69-156 des Actes, Actes Sud (Traduire Freud).

ATLAS (1991): $7^{\mathrm{e}}$ Assises, 9-11 novembre 1990, la retraduction (de Proust, de Dickens) - un des thèmes majeurs de ces Assises.

BENHAMOU, A. F. (1990) : «Quel langage pour le theâtre ? À propos de cinq traductions d'Othello», Palimpsestes, $\mathrm{n}^{\circ} 4$, pp. $9-32$

BERMAN, A. (1985) : «La traduction et la lettre - lointain», Les Tours de Babel, Mauvezin, TER, pp. 35-150.

BERMAN, A. (1990) : «La retraduction comme espace de traduction», Palimpsestes, n 4, pp. 1-7.

BOURGUIGNON et al. (1989): Traduire Freud, Paris, PUF, 380 p.

DROIT, R.-P. (1991) : «Comment traduire Hegel ?», Le Monde, 11 octobre 1991 (à propos de la retraduction de La phénoménologie de l'Esprit, par J.-P. Lefebvre).

GAMBIER, Y. (à paraître) : «L'é-change de langue», Actes du colloque Langage et Praxis, Montpellier, 24-26 mai 1990.

GAMBIER, Y. (1992) : «Adaptation : une ambiguitté à interroger», Meta, 37-3, Montréal, Presses de l'Université de Montréal, p. 573

GRESSET, M. (1990) : «Retraduire, (re)mettre en scène. L'exemple de Sanctuary (Faulkner)», Palimpsestes, $\mathrm{n}^{\circ} 4$, pp. 33-44.

Humanistic Dilemmas: Translation in the Humanities and Social Sciences, colloque tenu à l'Universite de New York a Binghamton, 26-28 septembre 1991 .

LADMIRAL, J.-R. (1979): Traduire : Théorèmes pour la traduction, Paris, Payot, 277 p.

MESCHONNIC, H. (1973): Pour la poétique II. Épistémologie de l'écriture poétique de la traduction, Paris, Gallimard (en particulier, pp. 305-454).

MESCHONNIC, H. (1981) : «Traduire la Bible, de Jonas à Jona», Langue française, n 51, pp. $35-52$.

Palimpsestes (1990): Retraduire (80 p.), Paris, Publications de la Sorbonne Nouvelle, $n^{\circ} 4$.

PASANEN, M. (1990) : «Survival in Translation», exposé au $12^{\mathrm{e}}$ Congrès mondial de la FIT, Belgrade.

RODRIGUEZ, L. (1990) : «Sous le signe de Mercure, la retraduction», Palimpsestes, $\mathrm{n}^{\circ}$ 4, pp. 63-80.

SCIALOM, M. (1989): «La traduction de la Divine Comédie, baromètre de sa réception en France?», RLC (Revue de littérature comparée), $\mathrm{n}^{\circ}$ 2, avril/juin 1991, pp. 197-207.

TOPIA, A. (1990): «Finnegans Wake : la traduction parasitée», Palimpsestes, n 4, pp. 45-63. 